\title{
Temporal Evolution of the Pressure Profile and Mode Behavior during Internal Reconnection Events in the MAST Spherical Tokamak
}

\author{
Hiroshi TOJO, Mikhall P. GRYAZNEVICH ${ }^{1)}$, Akira Ejiri, Alan SYKES ${ }^{1)}$, Yuichi TAKASE \\ Graduate School of Frontier Sciences, The University of Tokyo, Kashiwa 277-8561, Japan \\ 1) EURATOM/UKAEA Fusion Association, Culham Science Centre, OX14 3DB, UK
}

(Received 16 November 2007 / Accepted 6 February 2008)

\begin{abstract}
Mode behaviors including non-linear development and pressure profiles during internal reconnection events are clarified in the MAST spherical tokamak. In a $q_{0}>1$ discharge, the tearing mode is a trigger; however, non-linearity of modes is not confirmed. On the other hand, in a $q_{0}<1$ discharge, the harmonics of a $m / n=4 / 1$ mode of $\sim 22 \mathrm{kHz}$ are confirmed. Method for identification of poloidal mode in ST configuration is also given.
\end{abstract}

(c) 2008 The Japan Society of Plasma Science and Nuclear Fusion Research

Keywords: IRE, spherical tokamak, bicoherence, poloidal mode, reconnection

DOI: $10.1585 /$ pfr.3.S1065

\section{Introduction}

Internal reconnection events (IREs), which limit performance, have often been observed in spherical tokamaks (STs). IREs are MHD instabilities that show mode growth of precursor and flatten the pressure profile. In the worst case, they result in disruption. Three-dimensional MHD simulations have shown that linear growth rate and mode number of pressure-driven modes depend on $q_{0}$ (safety factor at the magnetic axis) and $\beta_{\mathrm{t}}$ (toroidal beta), and that non-linear coupling of linear modes leads to a large plasma deformation [1]. In this situation, magnetic reconnection occurs and core plasma energy is lost along the reconnected magnetic line. The relationships between the mode number (and its growth rate) and $q_{0}$ and $\beta_{\mathrm{t}}$ have not been clarified experimentally. Moreover, non-linear coupling of modes just before IRE have not been investigated in detail. In MAST [2], IREs appear in both $q_{0}>1$ and $q_{0}<1$ discharges and they often limit the achievable plasma beta in high- $\beta$ regimes. The objectives of this study are to clarify mode number and its dependence on such parameters, and to confirm the existence of non-linear coupling.

\section{Experimental Setup}

\subsection{Mirnov coil array and identification of poloidal mode number}

Mirnov coils (magnetic probes) are sensitive to external mode behavior and are employed to identify mode numbers. In MAST, magnetic coils are located along the toroidal direction (12ch at the most), along the center column (40ch), and along the outboard (18ch at the most). In STs, determination of poloidal mode numbers $(m)$ is not easy because of the effects of non-circular magnetic flux

author'se-mail: tojo@fusion.k.u-tokyo.ac.jp surfaces and asymmetric magnetic field pitch angle. To overcome these problems, we developed a method to evaluate the poloidal mode number and its intensity. First, we assume helical filamentary currents, which generate magnetic fluctuations, on their resonant surface calculated from EFIT [3]. The poloidal positions of these filaments are decided by tracing a magnetic field line. The number of alternating positive and negative filament pairs coincides with the mode number. Figure 1 shows trajectories of magnetic lines at the surfaces $q=1,1.5,2$, and 3 . In this case, the initial poloidal angle $(\theta=0)$ is located at the outboard midplane. The poloidal location of each filament (i.e., marker filament) can be obtained from the intersection of the curve and integer or half-integer toroidal turns (Fig. 1). The tangents of the lines become steep at $\theta \sim \pi$ because of the strong toroidal field at the inboard side. Therefore, the poloidal distribution of the filaments is asymmetric, and most of them tend to be located at the

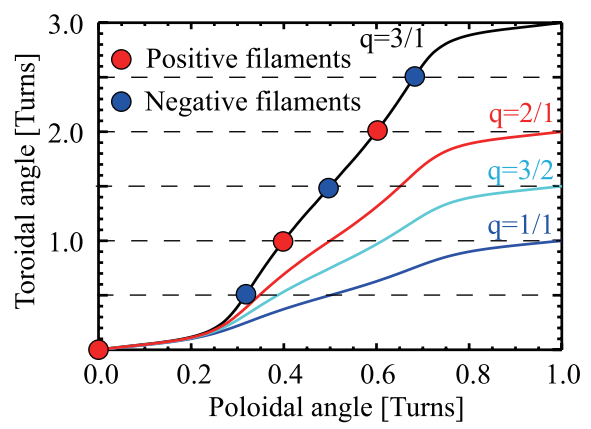

Fig. 1 Trajectory of the magnetic field lines at the rational surfaces $q=1,1.5,2$, and 3. Poloidal angles of filaments are obtained from the intersections between the traces and horizontal lines with intervals of half-integer turns. 


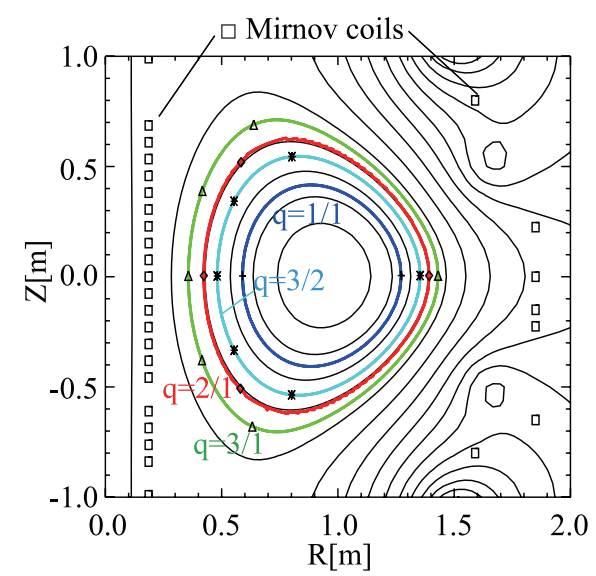

Fig. 2 Contour plot of the poloidal flux surfaces and filament positions at rational surfaces $q=1$ (cross), 1.5 (asterisk), 2 (rectangle), and 3 (triangle).

high field side (Fig. 2). The number of filaments (and their rational surface), current amplitude, and the poloidal location of the initial filament were obtained from the best fit to the measured Mirnov coil signals. By performing fittings at each timing, we can derive poloidal rotation and amplitude growth of the mode. However, in this report, we used band-passed signals and assumed single-mode distortion located at a rational surface to study the role of the dominant mode. Note that this model is appropriate for localized rational modes, such as tearing modes.

\subsection{Measurement of soft X-ray profile}

Soft X-ray radiation (SXR) is useful in the study of MHD phenomena, and it provides information on internal mode structure. Horizontal (32ch) and tangential (16ch) SXR cameras are employed, and their sight lines are shown in Fig. 3. To compare SXR behavior, $q$ (calculated from EFIT), and (electron) pressure profile (measured by Thomson scattering), the sight lines of each SXR channel are labeled by the minimum poloidal flux $(\psi)$ obtained by EFIT. In other words, along each sight line, we determine the minimum of $\psi$ (symbols in Fig. 3) and corresponding radial position $R$.

\subsection{Analysis using bispectrum to show non- linear coupling}

To clarify non-linear development of IRE precursors, we performed bispectrum analysis, which is a Fourier analysis for three-wave interaction and used frequently in turbulence analysis $[4,5]$. Generally, auto bispectrum is defined as

$$
B\left(f_{1}, f_{2}\right)=\left\langle X\left(f_{1}\right) X\left(f_{2}\right) X^{*}\left(f_{3}\right)\right\rangle,
$$

where $f_{3}=f_{1}+f_{2}$ and $X(f)$ is a Fourier components of signal $x(t)$. To evaluate the degree of nonlinear coupling for each frequency component, the following normalized

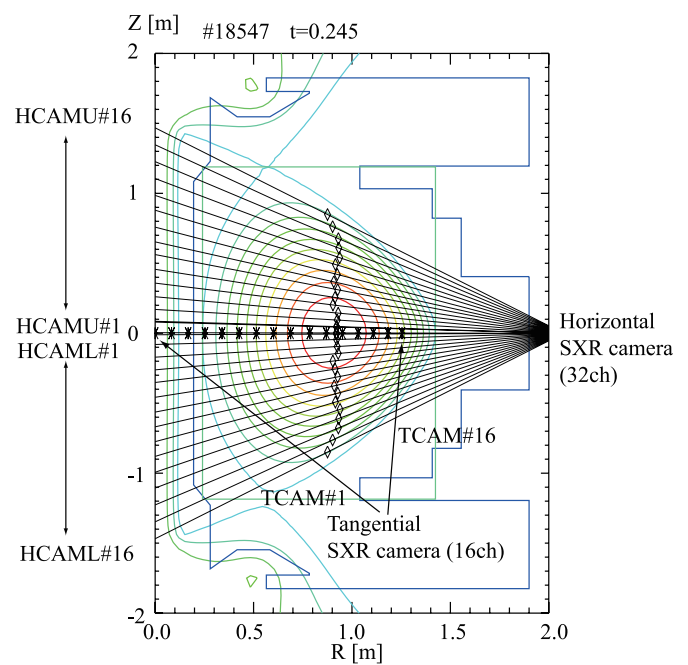

Fig. 3 Configuration of horizontal (sight lines) and tangential (asterisk) SXR cameras.

auto bispectrum is commonly used:

$$
b^{2}\left(f_{1}, f_{2}\right)=\frac{\left|B^{2}\left(f_{1}, f_{2}\right)\right|^{2}}{\left\langle\left|X\left(f_{1}\right) X\left(f_{2}\right)\right|^{2}\right\rangle\left\langle\left|X^{*}\left(f_{3}\right)\right|^{2}\right\rangle} .
$$

If there is a strong non-linear relationship between $f_{1}$ and $f_{2}, b^{2}$ becomes large (nearly 1 ). We analyzed Mirnov coil data for understanding non-linear development in IRE precursors. To obtain accurate bicoherence, a large number of ensembles should be averaged. In MAST, there are twelve (maximum) toroidal Mirnov coils around the center column at the same poloidal position. We employed these coil data to perform ensemble averaging. Total bicoherence is defined as

$$
B_{\mathrm{tot}}^{2}=\sum_{f_{1}, f_{2}} B^{2}\left(f_{1}, f_{2}\right),
$$

which represents quantitative measurement of non-linear coupling.

\section{IRE Discharges}

\subsection{IRE with $q_{0}>1$}

A typical discharge with $q_{0}>1$ and $\beta_{\mathrm{t}} \sim 9 \%$ is shown in Fig. 4. Increase in plasma current $\left(I_{\mathrm{p}}\right)$ and drop in the electron density, detected by an interferometer, were observed. The increase in the emission of $D_{\alpha}$ indicates interaction between the plasma and the vacuum vessel due to the loss of the plasma. One of the unique characteristics of the events is that the SXR radiation profile shows propagation of the drop starting from the area of fluctuations (precursor). This IRE has a precursor not only in SXR radiation but also in the Mirnov coil signals. The position of reconnection is close to HCAMU $\sharp 12(R \sim 1.25)$, because HCAMU $\sharp 13-15$ increases while HCAMU $\sharp 3-11$ decreases after $t \sim 0.2495$ (arrows in Fig. 4). We executed the fitting code to determine the mode number. Here, we applied 


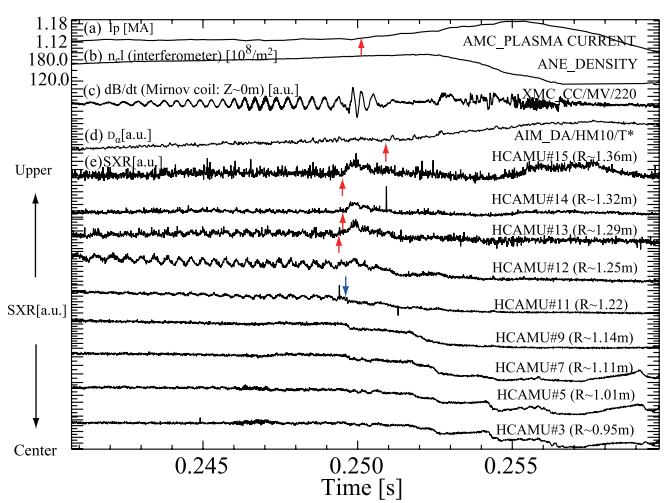

Fig. 4 IRE in discharge with $q_{0}>1$. (a): Plasma current [kA], (b): Line-integrated electron density $\left[/ \mathrm{m}^{2}\right](\mathrm{c}): \mathrm{d} B / \mathrm{d} t$ from Mirnov coil [a.u.], (d): Emission of $D_{\alpha}$ [a.u.] (e): SXR radiation [a.u.] on horizontal SXR camera from upper side to the center of the plasma.

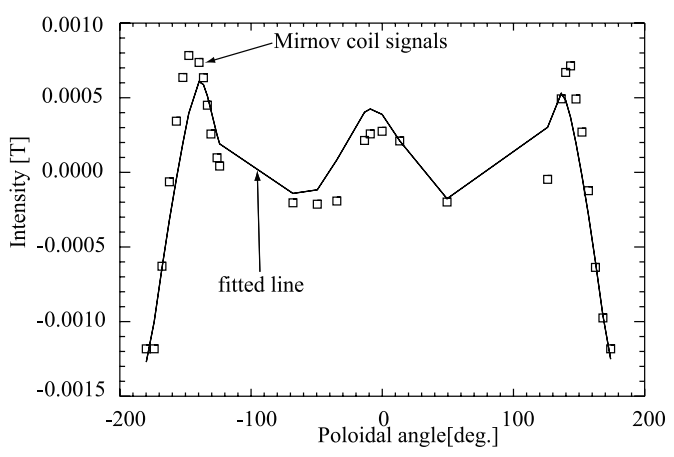

Fig. 5 Integrated Mirov coils signal (1-5 kHz) and fitted curve of the model assuming only $m / n=2 / 1$ mode for $\sharp 18547$ at $t=0.24785 \mathrm{~s}$. The origin of poloidal angle is the magnetic axis determined by EFIT.

band-pass (1-5 kHz) filtering to the time-integrated Mirnov signals. An example of the fitting is shown in Fig. 5. The squares represent the Mirnov coil data at $t=0.24785$ and the line shows a fitted curve assuming only $2 / 1$ mode (filaments) at the rational surface $q=2$. They show good agreement. Figure 6 indicates time evolution of the calculated helical filament current (a), poloidal angle of a filament (b), and fitting error (c). As described in 2.1, filament current reflects mode intensity, and it can be used to derive the growth time. From $t \sim 0.241$, the filament current increases exponentially, indicating linear growth. The time constant $\left(\tau_{\text {fil }}\right)$ of this growth is about $6 \mathrm{~ms}$. The phase of the filament decreases continuously, showing poloidal rotation of the filaments. The error of the fitting is represented by the normalized residual error $\chi^{2}$. When $\chi^{2}$ is less than 10 , the quality of the fitting is acceptable in the present analysis. We performed bispectrum calculation on the toroidal Mirnov signals $\left(\phi=10^{\circ}, 50^{\circ}, 70^{\circ}, 110^{\circ}, 130^{\circ}, 170^{\circ}, 230^{\circ}\right.$, and $290^{\circ}$ ) with $250 \mathrm{kHz}$ sampling rate in order to check non-linearity. The number of ensembles is 8 and the number of data points per ensemble is $750(3 \mathrm{~ms})$. The results

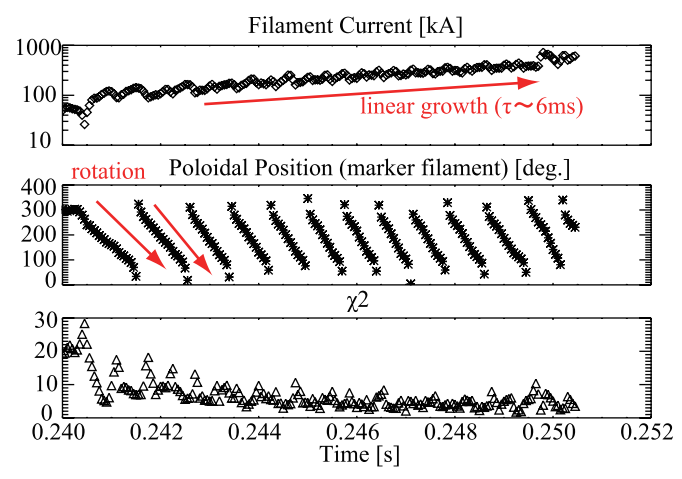

Fig. 6 Time evolution of the fitting parameters for $\sharp 18547$. (a): Filament current $[\mathrm{kA}](\mathrm{b})$ : Poloidal angle of the marker filament selected to understand poloidal rotation, and (c): $\chi^{2}$ (error of the fittings).

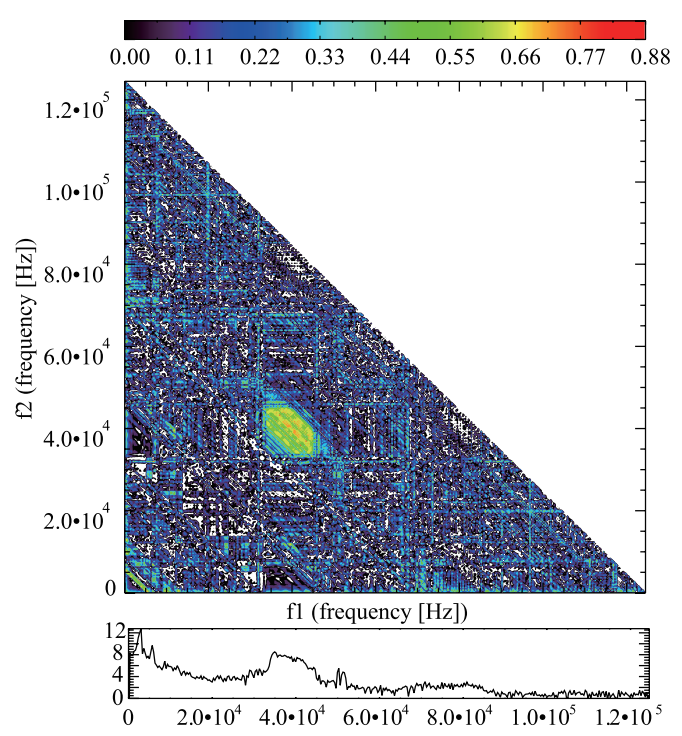

Fig. $7 \# 18547$ Bicoherence spectrum $(t=0.245-0.248)$ of the toroidal Mirnov coils (top) and power spectrum in the logarithmic scale (bottom).

are shown in Fig. 7. There is strong bicoherence around $\left(f_{1}, f_{2}\right) \sim(40 \mathrm{kHz}, 40 \mathrm{kHz})$, indicating harmonic coupling with itself; however, these modes are localized near the centre of the plasma $(R \sim 1.0 \mathrm{~m})$ observed in SXR signals and they seems to be independent of the $2 / 1$ mode. No strong bicoherence was observed around the frequency of the $2 / 1$ mode. The position of $q=2$ at $t=0.248 \mathrm{~s}$ from the EFIT result is $R \sim 1.33 \mathrm{~m}$, and it is not far from the expected reconnection position $(R \sim 1.25 \mathrm{~m})$ observed in SXR, but the high-frequency mode is observed at $R \sim 1.0 \mathrm{~m}$. Thus, the growth of the $2 / 1$ mode at $q=2$ seems to trigger IREs.

\subsection{IRE with $q_{0}<1$}

In the case of $q_{0}<1$, IRE should be distinguished from sawtooth oscillation, which is an instability at the $q=1$ rational surface. In this study, IRE is defined as the 


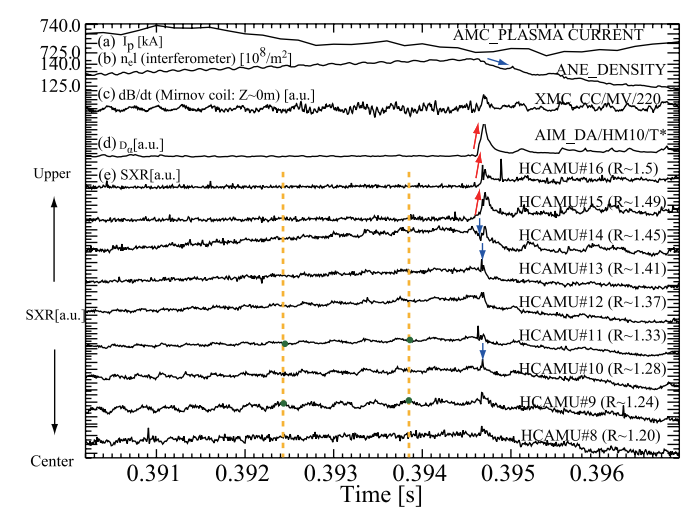

Fig. 8 IRE in a discharge with $q_{0}<1$. (a): Plasma current [kA], (b): Line-integrated electron density $\left[/ \mathrm{m}^{2}\right](\mathrm{c}): \mathrm{d} B / \mathrm{d} t$ from Mirnov coil [a.u.], (d): Emission of $D_{\alpha}$ [a.u.], and (e): SXR radiation [a.u.] on horizontal SXR camera from top to center (SXR signals for midplane to upper chords) of the plasma. Yellow dotted line shows significant phase difference between the two SXR signals (HCAMU $\sharp 9$ and $\sharp 11$ ).

instability with mode couplings and/or is often accompanied by a collapse at the rational surface, except for the $q=1$ surface. Figure 8 shows an IRE discharge with $q_{0}<1$. This IRE does not terminate the plasma but reduces $\beta_{\mathrm{t}}$ significantly. The position of the reconnection is around HCAMU $\sharp 14(R \sim 1.45 \mathrm{~m})$. However, the area with strong oscillation is close to HCAMU $\sharp 9-11(R \sim 1.2-1.3 \mathrm{~m})$, and the phase difference between HCAMU $\sharp 9$ and 11 is significant (two yellow lines in Fig. 8). Tearing modes create islands, where pressure and other values tend to become flat. When an island rotates along the toroidal or poloidal direction, the local gradient oscillates. If SXR is measured on both the sides, it will show opposite behaviors. Thus, the out-of-phase oscillations between close SXR chords indicate the existence of an island. Therefore, this phase change is caused by a tearing mode, and the resonant surface is at $R \sim 1.24-1.28 \mathrm{~m}$. The fitting code does not work well on this shot because the calculated position of the resonant surface (from EFIT) is presumably not good. However, as indicated in section 2.1, the number of filaments (model of the mode) on the outboard side is very low because of the low aspect ratio configuration. Therefore, the poloidal mode number can be estimated roughly by counting peaks along the centre stack and adding one for outboard filament. In this manner, $m / n=2 / 1$ is confirmed for this $q_{0}<1$ discharge. Note that it is difficult to derive the growth time and rotation w/o the fitting. The width of the $2 / 1$ mode appears to increase, as shown by SXR radiation from the outer side, e.g., HCAMU $\sharp 14$ from $t \sim 0.392 \mathrm{~s}$. In addition, Mirnov coils show another high-frequency mode $(\sim 22 \mathrm{kHz})$. Unfortunately, there is no strong cross-correlation between this high-frequency mode and any SXR channel, and the position of this mode is not clear from SXR signals. How-

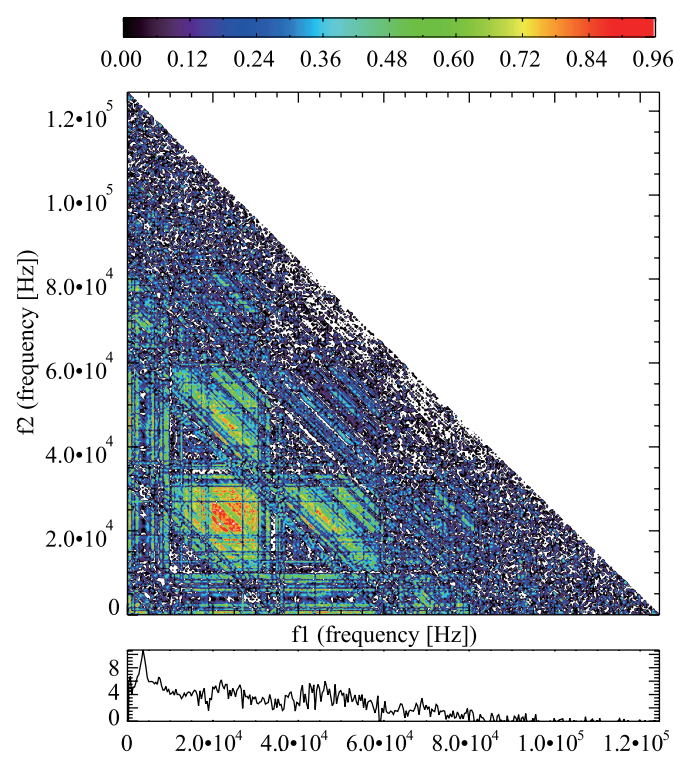

Fig. $9 \sharp 18501$ Bicoherence spectrum $(t=0.389-0.392)$ of the toroidal Mirnov coils (top) and power spectrum on logarithmic scale (bottom).

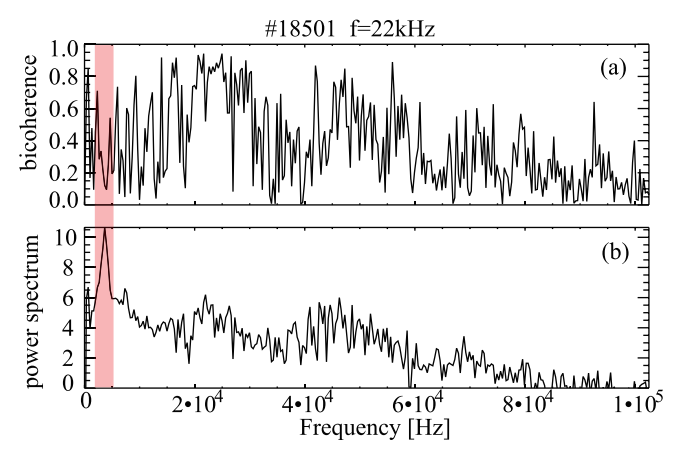

Fig. $10 \sharp 18501$ Bicoherence spectrum $(t=0.389-0.392)$ at $f_{2}=$ $22 \mathrm{kHz}$ (a) and power spectrum in logarithmic scale (b). The red shaded area is the $2 / 1$ mode component.

ever, the poloidal mode number $(m)$ is presumed to be 4 and the toroidal mode $(n)$ is 1 . These mode numbers are determined by the Mirnov coil arrays. Therefore, this mode localizes at the edge $(R>1.5 \mathrm{~m})$. To clarify the relationship between these modes (i.e., 3 and $22 \mathrm{kHz}$ ), the bicoherence spectrum is calculated (Fig. 9). For $t=0.389-0.392$, there is no significant physical coupling between the two modes. Figure 10 shows the bicoherence for $f_{2}=22 \mathrm{kHz}$ as a function of $f_{1}$. The power spectrum is also plotted at the bottom. No strong bicoherence was found around $3 \mathrm{kHz}$ (red shaded area), indicating no coupling between the 2/1 and 4/1 modes from this analysis. Instead, the 4/1 mode $(\sim 22 \mathrm{kHz})$ shows second and third harmonic modes (approximately 45 and $68 \mathrm{kHz}$ ). Figure 11 shows the result in a later period $t=0.392-0.395 \mathrm{~s}$. These frequencies are localized on the frequency space and the sharp coherence disappears, but broad bicoherence appears in the region $f_{1}+f_{2}<50 \mathrm{kHz}$, suggesting non-linear coupling among 


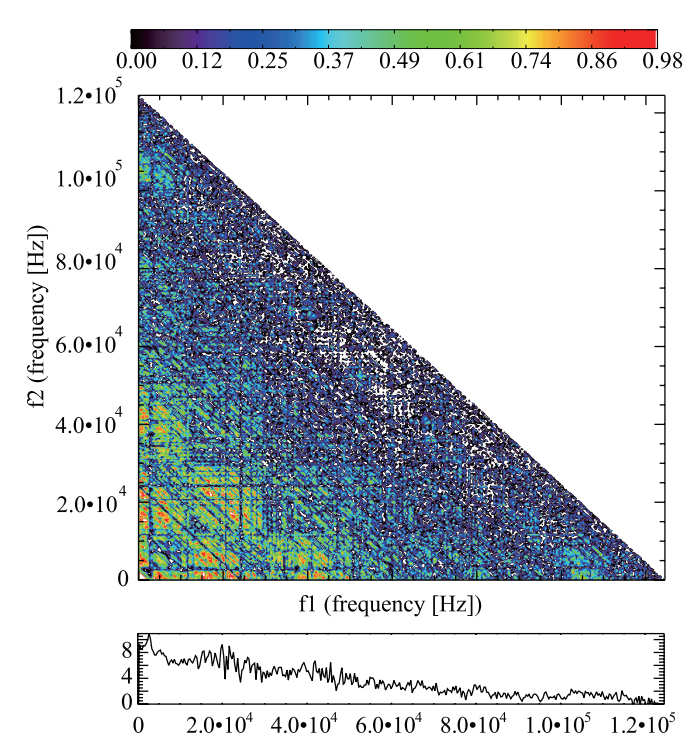

Fig. $11 \sharp 18501$ Bicoherence spectrum $(t=0.392-0.395)$ of the toroidal Mirnov coils (top) and power spectrum on logarithmic scale (bottom).

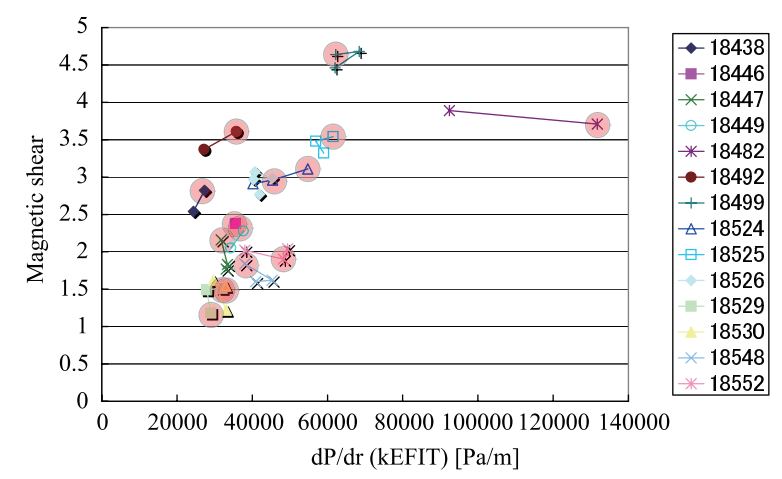

Fig. 12 Plot of magnetic shear $(S)$ and pressure gradient from the kEFIT result at the $q=2$ rational surface before $(0$ $21 \mathrm{~ms}$ ) IRE. The symbols correspond to the specitic shots. The red shaded circles indicate values just before IRE.

a large number of modes. This is consistent with the nonlinear phase of the MHD simulation in reference [1]. The total squared bispectrum (duration of ensemble is $\sim 3 \mathrm{~ms}$ ) at these two periods shows a significant increase of about 50 times. However, we cannot decide which mode is a trigger of this IRE, because the position of the high-frequency mode was not identified.

\section{IRE Drive}

To understand the drive of IRE is important for its prevention. For comparing with reference [1], which suggested a pressure-driven mode as the source of the insta- bilities, we evaluated the pressure gradient for some IRE discharges. Figure 12 shows time evolutions of a correlation between magnetic shear $(S)$ and pressure gradient $(\mathrm{d} P / \mathrm{d} r)$ for thirteen shots, where magnetic shear were defined as $S=(r / q) \cdot \mathrm{d} q / \mathrm{d} r$. These two parameters are calculated by kEFIT, which takes into account the pressure profile obtained from the Thomson scattering profile [6]. Red circles represent the values just before each IRE. IRE occurs when the pressure gradient exceeds the critical value, which becomes high at high shear. Note that although there are several shots showing an increase in the gradient by the time of IRE, some shots do not show such behavior under the result from kEFIT. Therefore, it is possible that other hidden parameters control the growth of IREs.

\section{Summary and Conclusion}

In this paper, IREs in the MAST plasma were studied using a filament model, in which low aspect ratio effects are taken into account. In the case of $q_{0}>1$, no mode coupling related to IRE is observed, but the growth of the $2 / 1$ tearing mode with $\tau \sim 6 \mathrm{~ms}$ is confirmed. The mode triggers a collapse. In the IRE with $q_{0}<1$, slow growth of the $2 / 1$ tearing mode is observed and the second and third harmonics from a $4 / 1$ mode, $\left(f_{1}, f_{2}\right) \sim(22 \mathrm{kHz}, 22 \mathrm{kHz})$ and $\left(f_{1}, f_{2}\right) \sim(22 \mathrm{kHz}, 44 \mathrm{kHz})$, are confirmed in Mirnov coil signals. After that, these bicoherence peaks disappear and broad bicoherence in the range of $f_{1}+f_{2}<50 \mathrm{kHz}$ is observed. This is consistent with the time evolution of each mode in [1]. Although we have not found a clear evidence of non-linear development causing an IRE followed by collapse, the $2 / 1$ mode may trigger the collapse. To find out the source of IRE, we compare time evolution of pressure gradient and magnetic shear. Many IREs show the existence of a critical pressure gradient, which increases with magnetic shear at $q=2$, suggesting a pressure-driven nature. However, some shots do not show such behavior, and it is necessary to find other parameters determining the growth of such IREs.

\section{Acknowledgment}

This work was supported by JSPF Grant-in-Aid for Scientific Research 16106013.

[1] N. Mizuguchi et al., Phys. Plasmas 7, 940 (2000).

[2] B. Lloyd et al., Nucl. Fusion 43, 1665 (2003).

[3] L. Lao et al., Nucl. Fusion 25, 1611 (1985).

[4] Y.C. Kim and E.J. Powers, Phys. Fluids 21, 1452 (1978).

[5] Y. Nagashima et al., Plasma Phys. Control. Fusion 49, 1611 (2007).

[6] L.C. Appel et al., Nucl. Fusion 41, 169 (2001). 